\title{
Thermodynamic Casimir forces in strongly anisotropic systems within the $N \rightarrow \infty$ class
}

\author{
M. Łebek $^{1^{*}}$, P. Jakubczyk ${ }^{1}$, \\ 1 Institute of Theoretical Physics, Faculty of Physics, University of Warsaw, Pasteura 5, 02-093 \\ Warsaw, Poland \\ *m.lebek@student.uw.edu.pl
}

September 6, 2021

\begin{abstract}
We analyze the thermodynamic Casimir effect in strongly anizotropic systems from the vectorial $N \rightarrow \infty$ class in a slab geometry. Employing the imperfect (mean-field) Bose gas as a representative example, we demonstrate the key role of spatial dimensionality $d$ in determining the character of the effective fluctuation-mediated interaction between the confining walls. For a particular, physically conceivable choice of anisotropic dispersion and periodic boundary conditions, we show that the Casimir force at criticality as well as within the low-temperature phase is repulsive for dimensionality $d \in\left(\frac{5}{2}, 4\right) \cup(6,8) \cup(10,12) \cup \ldots$ and attractive for $d \in(4,6) \cup(8,10) \cup \ldots$ We argue, that for $d \in\{4,6,8 \ldots\}$ the Casimir interaction entirely vanishes in the scaling limit. We discuss implications of our results for systems characterized by $1 / N>0$ and possible realizations in the context of quantum phase transitions.
\end{abstract}

\section{Contents}

1 Introduction 2

2 The mean-field Bose gas 3

2.1 Summary of the bulk solution

3 Saddle-point equation 5

4 Excess grand canonical free energy

5 Discussion and outlook 9

A The remainder term $\mathcal{R}_{1}^{(1)}$

B Properties of $F$ and $G$ functions

\begin{tabular}{ll} 
References & 12 \\
\hline
\end{tabular} 


\section{Introduction}

The thermodynamic Casimir effect received substantial interest over the last years, [1-5] both from theoretical and experimental points of view. At the heart of the theory underlying this phenomenon lies the concept of the dimensionless scaling function $\Delta(x)$, describing the variation of the excess free energy density $\omega_{s}$ upon changing the scaling variable $x \sim D / \xi$, where, in the presently considered setup of a slab geometry, $D$ is the system extension in one of the directions, while $\xi$ denotes the correlation length. The (linear) system size $L$ in the remaining directions is assumed infinite $(L / D \rightarrow$ $\infty)$. The excess free energy density $\omega_{s}$ is generically related to $\Delta(x)$ via

$$
\omega_{s}=k_{B} T \frac{\Delta(x)}{D^{d-1}}
$$

in the so-called scaling limit, where both $D$ and $\xi$ are large as compared to microscopic scales. Here $d$ is the system dimensionality and $\Delta(x)$ is universal in the sense that it depends on the bulk universality class and the boundary conditions, but not fine microscopic details of the system. The Casimir force (per unit area) is given by $F=-\frac{\partial \omega_{s}}{\partial D}$. The scaling function $\Delta(x)$ was computed for a variety of systems within exact and approximate analytical approaches as well as numerical simulations. It was also measured experimentally (see Ref. [5] for a recent review).

There are few known cases, where Eq. (1) does not apply. One such situation arises in systems exhibiting strongly anisotropic scale invariance, [6] where the singularity of the correlation function at the phase transition is related to (at least) two correlation lengths $\xi_{\|}$and $\xi_{\perp}$ diverging with different critical exponents so that $\xi_{\perp} \sim \xi_{\|}^{\theta_{A}}$, and the anisotropy exponent $\theta_{A} \neq 1$. As was demonstrated in Ref. [7], the Casimir energy decay exponent $\zeta_{0}=d-1$ in Eq. (1) becomes in such a situation modified. This is interesting, because, for dimensional reasons, a quantity of dimension [length] must then appear in the corresponding expression for $\omega_{s}$. This in turn may originate only from the microscopic quantities, thus restricting the universal character of the Casimir interaction. Specifically, for the so-called $m$-axial Lifshitz point, [8,9] Ref. [7] predicts that Eq. (1] becomes replaced by

$$
\omega_{s}=k_{B} T \frac{\Gamma \Delta_{m}^{d}(x)}{D^{\zeta_{m}}}
$$

where

$$
\zeta_{m}=\frac{d-m}{\theta_{A}}+m-1,
$$

and $\Gamma$ is a dimensionful scale factor, deriving from microscopic length scales and therefore nonuniversal. Equations (2) and (3) apply to the setup, where the confining walls are oriented perpendicular to one of the $m(m \leq d)$ directions, where the inverse propagator deviates from the standard quadratic form and is (up to anomalous dimensions) quartic in momentum.

In this paper we argue that Eq. (2) and (3) do not hold for the case of $N \rightarrow \infty$ universality class with $m=1$ and periodic boundary conditions in even dimensionalities $d=2 n, n \in\{2,3,4, \ldots\}$. We consider a microscopic model being a representative of this universality class and analyze the properties of the scaling function $\Delta_{m}^{d}(x)$ upon varying dimensionality $d$. By an exact analysis we demonstrate in particular that $\Delta_{1}^{d}(x)$ changes sign for each $d=2 n$ (and is identically equal zero for $d \in\{4,6,8, \ldots\})$. In consequence, the corresponding Casimir interaction is repulsive for $d \in$ $\left(\frac{5}{2}, 4\right) \cup(6,8) \cup \ldots$ and attractive for $d \in(4,6) \cup(8,10) \cup \ldots$ This is in stark contrast to the case of isotropic systems with quadratic dispersion, where (for periodic boundary conditions) the Casimir force is always attractive (in any dimensionality and also for the entire family of $O(N)$ universality classes), which is guaranteed by exact statements. [10,11] We clarify the character of the Casimir 
interaction in the peculiar case of $d \in\{4,6,8, \ldots\}$ by demonstrating that there is no subdominant contribution to the excess free energy, surviving the scaling limit. By continuity in $1 / N$ we argue, that (at least for some values of the scaling variable $x$ ) the sign of the Casimir force also changes at particular (presumably non-integer) values of $d$ provided $1 / N>0$ is sufficiently small.

A substantial technical part of our analysis heavily relies on an earlier calculation presented in Ref. [12]. In that paper we confirmed the predictions summarized in Eq. (2) and Eq. (3) and calculated the scaling function focusing mainly on spatial dimensionalities corresponding to $d=3$. As we demonstrate in the present analysis, varying dimensionality has a drastic and unexpected impact on the emergent physical picture. In order to avoid repetitions, we will frequently refer to Ref. [12] throughout the paper.

The present calculation is exact and is carried out for the imperfect Bose gas, which constitutes a particular microscopic representative of the $N \rightarrow \infty$ universality class. More precisely, as established in Ref. [13] (for the isotropic case at least), the imperfect Bose gas is equivalent to the $O(2 N)$ model in the limit $N \rightarrow \infty$ and the corresponding scaling functions [14, 15] for Casimir energy differ by a global factor of two. We expect a similar correspondence to hold for the anisotropic situation as well.

In Sec. II we discuss the model and summarize the relevant elements of its thermodynamics. Sec. III contains an analysis of the saddle-point equation. Both Sec. II and Sec. III strongly rely on Ref. [12]. However, to drag the correct conclusions it is necessary to keep track or remainder terms (vanishing in the scaling limit) which constitutes the important extension of Ref. [12]. All new, physically relevant results are contained in Sec. IV, where we analyze the excess free energy varying dimensionality. Sec. V contains a summary and a portion of technical details of the analysis is postponed to appendices.

\section{The mean-field Bose gas}

The Bose-Einstein condensation in the imperfect (mean-field) Bose gas [13,16-21] is a representative of a bulk phase transition within the $N \rightarrow \infty$ universality class. The corresponding Hamiltonian is given by

$$
\hat{H}=\sum_{\mathbf{k}} \epsilon_{\mathbf{k}} \hat{n}_{\mathbf{k}}+\frac{a}{2 V} \hat{N}^{2}
$$

The repulsive mean-field interaction term $\hat{V}_{m f}=\frac{a}{2 V} \hat{N}^{2}(a>0)$ arises from the long-range repulsive part $v(r)$ of a 2-particle interaction potential in the Kac $\operatorname{limit}_{\gamma \rightarrow 0} \gamma^{d} v(\gamma r)$, corresponding to vanishing interaction strength and diverging range. We will consider the anisotropic setup, where

$$
\epsilon_{\mathbf{k}} \rightarrow \tilde{\epsilon}_{\mathbf{k}}=\sum_{i=1}^{d-m} t_{0}\left(k_{i} A\right)^{2}+\sum_{i=d-m+1}^{d} t\left(k_{i} A\right)^{4}
$$

We also introduce

$$
\tilde{\epsilon}_{k_{1}}=t_{0}\left(k_{1} A\right)^{2} \quad \text { and } \quad \tilde{\epsilon}_{k_{d}}=t\left(k_{d} A\right)^{4} .
$$

for future reference. The properties of $\epsilon_{\mathbf{k}}$ may be inherited from the underlying lattice structure. [22] Note that only the asymptotic behavior at small wavevector $\mathbf{k}$ is relevant for the critical singularities and therefore in Eq. (5) we replace the entire dispersion with its low-momentum asymptotic form (denoted $\tilde{\epsilon}_{\mathbf{k}}$ ). We assume $t_{0}>0, t>0$. The quantity $A$ is a microscopic length, which may be identified with a lattice constant. The bosonic particles are assumed spinless for simplicity. The system is $d$-dimensional and is enclosed in volume $V=L^{d-1} D$, where $L \gg D \gg l_{\text {mic }}$ and $l_{\text {mic }}$ denotes 
all the microscopic length scales present in the system. The quantity $D$ measures the system extension in the $d$-th direction along which the dispersion is quartic [see Eq. (5)]. We work within the framework of the grand canonical ensemble. The corresponding grand canonical partition function may be written as [19]

$$
\Xi(L, D, \mu, T)=-i \exp \left(\frac{\beta V}{2 a} \mu^{2}\right) \sqrt{\frac{V}{2 \pi \beta a}} \int_{\beta \alpha-i \infty}^{\beta \alpha+i \infty} \mathrm{d} s \exp [-V \varphi(s)] .
$$

The parameter $\alpha<0$ is arbitrary, $\beta^{-1}=k_{B} T$ and

$$
\varphi(s)=\frac{1}{\beta a}\left(-\frac{s^{2}}{2}+s \beta \mu\right)-\frac{1}{V} \log \Xi_{0}\left(\frac{s}{\beta}, T\right) .
$$

with the quantity $\Xi_{0}\left(\frac{s}{\beta}, T\right)$ denoting the grand canonical partition function of the noninteracting Bose gas [23] evaluated at chemical potential $\mu=\frac{s}{\beta}$ and temperature $T$. The presence of the volume factor in the term $\exp [-V \varphi(s)]$ in Eq. (7) guarantees that the saddle point analysis of Eq. (7) becomes exact for $V \rightarrow \infty$ (i.e. $L \rightarrow \infty$ ). The excess grand-canonical free energy density

$$
\omega_{s}(D, \mu, T)=\lim _{L \rightarrow \infty}\left[\frac{\Omega(L, D, T, \mu)}{L^{d-1}}-D \omega_{b}(T, \mu)\right],
$$

is related to the Casimir force (per unit area) $F(D, \mu, T)$ via

$$
F(D, \mu, T)=-\frac{\partial \omega_{s}(D, \mu, T)}{\partial D} .
$$

The grand-canonical free energy is evaluated as $\Omega(L, D, T, \mu)=-\beta^{-1} \ln \Xi(T, L, D, \mu)$ and the bulk free energy density $\omega_{b}(T, \mu)$ is given by $\omega_{b}(T, \mu)=\lim _{L \rightarrow \infty} \frac{1}{L^{d}} \Omega(L, D=L, T, \mu)$. Using Eq. (7), we write the excess contribution to the grand potential as

$$
\omega_{s}(D, \mu, T)=\lim _{L \rightarrow \infty} \beta^{-1} D\left[\varphi(\bar{s})-\varphi_{b}\left(s_{0}\right)\right]
$$

where

$$
\varphi(\bar{s})=-\frac{\bar{s}^{2}}{2 a \beta}+\frac{\mu \bar{s}}{a}-\frac{1}{V}\left[\sum_{\mathbf{k} \neq\left(\mathbf{0}, k_{d}\right)} \sum_{r=1}^{\infty} \frac{1}{r} e^{r\left(\bar{s}-\beta \tilde{\mathbf{\epsilon}}_{\mathbf{k}}\right)}-\sum_{k_{d}} \log \left(1-e^{\bar{s}-\beta \tilde{\epsilon}_{k_{d}}}\right)\right],
$$

$\bar{s}$ denotes the solution to the saddle-point equation $\varphi^{\prime}(\bar{s})=0$, while $s_{0}$ corresponds to $\bar{s}$ in the bulk case (i.e. when $D=L$ and $L \rightarrow \infty$ ) and $\varphi_{b}(s)=\lim _{D \rightarrow L} \varphi(s)$. The strategy of the subsequent analysis amounts to solving $\varphi^{\prime}(\bar{s})=0$ at finite $D$ and evaluating Eq. (11) for the obtained value of $\bar{s}$.

\subsection{Summary of the bulk solution}

Below we recall the relevant features of the system in the thermodynamic limit, where $D=L \rightarrow \infty$ (see Refs. [12, 22]). Due to the anisotropic nature of the system, there are two characteristic length scales

$$
\lambda_{1}=2 A \sqrt{\pi} \sqrt{\beta t_{0}} \quad \lambda_{2}=A \frac{\pi}{\Gamma(5 / 4)}(\beta t)^{1 / 4},
$$

related to temperature and playing roles analogous to the thermal de Broglie length in the isotropic case. For convenience, some numerical factors are absorbed in the above definitions. We also introduce the 'thermal volume' parameter:

$$
V_{T}=\lambda_{1}^{d-m} \lambda_{2}^{m} .
$$


Analysis of the saddle-point equation in the thermodynamic limit [12, 22] leads to the following expression the critical line

$$
\mu_{c}(T)=\frac{a}{V_{T}} \zeta\left(\frac{1}{\psi}\right)
$$

where $\zeta$ denotes the Riemann zeta function and

$$
\frac{1}{\psi}=\frac{d}{2}-\frac{m}{4}
$$

It follows that the critical line obeys the universal power law $\mu_{c}(T) \sim T^{1 / \psi}$. The condensed phase is stable for $\mu>\mu_{c}(T)$ provided $\frac{1}{\psi}>1$. The condition $\frac{1}{\psi}=1$ determines the lower critical dimension $d_{l}(m)$ of the system. Note that for the usual isotropic case $(m=0)$ one recovers $d_{l}=2$, for the 'uniaxial' case $(m=1) d_{l}=\frac{5}{2}$, and the largest conceivable value of $d_{l}$ corresponds to $m=d$, where one obtains $d_{l}=4$. As shown in Ref. [22], the universal bulk properties of the system with given $m$ are closely related to the usual isotropic case in effective dimensionality $d_{\mathrm{eff}}=\frac{2}{\psi}$. This in turn can be related to the spherical [24] (Berlin-Kac) universality class, or the $N \rightarrow \infty$ limit of the $O(N)$ models. [25] This correspondence is restricted to bulk properties.

\section{Saddle-point equation}

The saddle-point equation $\varphi^{\prime}(\bar{s})=0$ can be cast in the following form [12]

$$
\begin{aligned}
\zeta\left(\frac{1}{\psi}\right)\left(-\frac{\bar{s}}{\mu_{c} \beta}+\varepsilon\right) & =g_{\frac{1}{\psi}}\left(e^{\bar{s}}\right)-\zeta\left(\frac{1}{\psi}\right)+\frac{1}{\Gamma(5 / 4)} \mathcal{R}_{1}^{(1)}+\frac{\Gamma(5 / 4)^{\frac{4}{\psi}-5}}{\pi^{\frac{4}{\psi}-4}}\left(\frac{\lambda_{2}}{D}\right)^{\frac{4}{\psi}-4} \sigma^{\frac{4}{\psi}-4} \sum_{n=1}^{\infty} F_{\frac{1}{\psi}}(n \sigma)+ \\
& -\frac{V_{T}}{V} \sum_{\mathbf{k}_{d}} \frac{1}{1-e^{\beta \tilde{\mathbf{k}}_{d}-\bar{s}}},
\end{aligned}
$$

where $g_{n}(z)=\sum_{k=1}^{\infty} \frac{z^{k}}{k^{n}}$ denotes the Bose function, $\varepsilon=\frac{\mu-\mu_{c}}{\mu_{c}}$, while

$$
\sigma=\frac{\pi}{\Gamma(5 / 4)} \frac{D}{\lambda_{2}}|\bar{s}|^{1 / 4} \quad F_{\kappa}(x)=\int_{0}^{\infty} \mathrm{d} p \frac{e^{-p}}{p^{\kappa}} \phi\left(x / p^{1 / 4}\right)
$$

and

$$
\phi(k)=\int_{-\infty}^{\infty} \mathrm{d} x e^{i k x} e^{-x^{4}}
$$

is the Fourier transform of the quartic Gaussian. Eq. (17) is exact and valid in an arbitrary thermodynamic state, for any $D$ and $L \gg D$. The remainder term $\mathcal{R}_{1}^{(1)}$ arises from application of the Euler-Maclaurin formula [12] and, importantly, can be dropped for $L \rightarrow \infty$ (even at $D$ finite). We provide an analysis of this term in the appendix A. Upon neglecting $\mathcal{R}_{1}^{(1)} \mathrm{Eq}$. 17 becomes equivalent to Eq. (42) of Ref. [12]. We continue by introducing the scaling variable

$$
x= \begin{cases}\varepsilon\left(\frac{D}{\lambda_{2}}\right)^{\frac{4}{\psi}-4}, & 1<\frac{1}{\psi}<2 \\ \varepsilon\left(\frac{D}{\lambda_{2}}\right)^{4}, & \frac{1}{\psi}>2,\end{cases}
$$

the sign of which is positive below bulk $T_{c}$ and negative otherwise. We do not analyze the case $\frac{1}{\psi}=2$ (corresponding to the upper critical dimension of the bulk transition) where $|\bar{s}|$ acquires logarithmic 
corrections. Note that $x \sim\left(D / \xi_{\|}\right)^{\gamma}$ with $\gamma=\frac{4}{\psi}-4$ for $1<\frac{1}{\psi}<2$ and $\gamma=4$ for $\frac{1}{\psi}>2$. The saddle-point equation is finally cast in the following convenient form:

$$
\begin{aligned}
\zeta\left(\frac{1}{\psi}\right) x= & \left(g_{\frac{1}{\psi}}\left(e^{\bar{s}}\right)-\zeta\left(\frac{1}{\psi}\right)+\frac{1}{\Gamma(5 / 4)} \mathcal{R}_{1}^{(1)}+\zeta\left(\frac{1}{\psi}\right) \frac{\bar{s}}{\beta \mu_{c}}\right)\left(\frac{D}{\lambda_{2}}\right)^{4\left(\frac{1}{\psi}-1\right)}+ \\
& \frac{\Gamma(5 / 4)^{\frac{4}{\psi}-5}}{\pi^{\frac{4}{\psi}-4}} \sigma^{\frac{4}{\psi}-4} \sum_{n=1}^{\infty} F_{\frac{1}{\psi}}(n \sigma)+\frac{V_{T}}{V}\left(\frac{D}{\lambda_{2}}\right)^{\frac{4}{\psi}-4} \sum_{\mathbf{k}_{d}} \frac{1}{e^{\beta \tilde{\mathbf{k}}_{d}-\bar{s}}-1}
\end{aligned}
$$

for $\frac{1}{\psi}<2$ and

$$
\begin{aligned}
\zeta\left(\frac{1}{\psi}\right) x= & \left(g_{\frac{1}{\psi}}\left(e^{\bar{s}}\right)-\zeta\left(\frac{1}{\psi}\right)+\frac{1}{\Gamma(5 / 4)} \mathcal{R}_{1}^{(1)}+\zeta\left(\frac{1}{\psi}\right) \frac{\bar{s}}{\beta \mu_{c}}\right)\left(\frac{D}{\lambda_{2}}\right)^{4}+ \\
& \frac{\Gamma(5 / 4)^{\frac{4}{\psi}-5}}{\pi^{\frac{4}{\psi}-4}}\left(\frac{\lambda_{2}}{D}\right)^{\frac{4}{\psi}} \sigma^{\frac{4}{\psi}-4} \sum_{n=1}^{\infty} F_{\frac{1}{\psi}}(n \sigma)+\frac{V_{T}}{V}\left(\frac{D}{\lambda_{2}}\right)^{4} \sum_{\mathbf{k}_{d}} \frac{1}{e^{\beta \tilde{\mathbf{k}}_{d}-\bar{s}}-1}
\end{aligned}
$$

for $\frac{1}{\psi}>2$. Note that (up to the remainder terms) Eq. 21 , $\sqrt{22}$ correspond do Eq. (44) and (45) of Ref. ( [12]). We shall now exclusively focus on $x \geq 0$, corresponding to $T \leq T_{c}$, where the Casimir interaction is expected to be long-ranged. After the above rearrangements, the scaling variable $x$ appears only on the left-hand side of the saddle point equation. At and below criticality $\bar{s} \rightarrow 0^{-}$and we may expand the right-hand side of the saddle-point equation for $|\bar{s}| \ll 1$.

We now consider the scaling limit, where $\frac{D}{\lambda_{2}} \gg 1$ and $\varepsilon \ll 1$, while the scaling variable $x$ may take any arbitrary nonnegative value. Recall also that $L \gg D$. In this limit, Eq. (21) takes the following form:

$$
\zeta\left(\frac{1}{\psi}\right) x=\left(\frac{\Gamma(5 / 4)}{\pi}\right)^{\frac{4}{\psi}-4} \sigma^{\frac{4}{\psi}-4}\left[\Gamma\left(1-\frac{1}{\psi}\right)+\frac{1}{\Gamma(5 / 4)} \sum_{n=1}^{\infty} F_{\frac{1}{\psi}}(n \sigma)\right]+\frac{V_{T}}{V|\bar{s}|}\left(\frac{D}{\lambda_{2}}\right)^{\frac{4}{\psi}-4}+\text { H.O.T. },
$$

while Eq. 22, can be written as

$$
\zeta\left(\frac{1}{\psi}\right) x=-\left(\frac{\Gamma(5 / 4)}{\pi}\right)^{4} \sigma^{4}\left[\frac{\zeta\left(\frac{1}{\psi}\right)}{\mu_{c} \beta}+\zeta\left(\frac{1}{\psi}-1\right)\right]+\frac{V_{T}}{V|\bar{s}|}\left(\frac{D}{\lambda_{2}}\right)^{4}+\text { H.O.T. . }
$$

Here 'H.O.T' stands for terms of higher order in $\bar{s}$ and $\frac{D}{L}$, which do not survive the analyzed limit.

In both the above cases, the left-hand side (LHS) is positive and does not depend on $|\bar{s}|$. The first term on the right-hand side (RHS) of Eq. (24) is manifestly negative. This implies that the second term (involving $V$ ) must give a finite contribution in the scaling limit to assure the existence of a solution. In consequence, $|\bar{s}|$ is of order $O\left(\frac{D^{4}}{V}\right)$ (assuming the microscopic length scales are of order 1). The situation is similar for Eq. 23 provided $\frac{1}{\psi} \geq \frac{7}{4}$ (for $m=1$ this corresponds to $d \geq 4$ ). We now focus on this case (considering that the opposite situation was analyzed in Ref. [12]) and inspect Eq. 23. Clearly $\Gamma\left(1-\frac{1}{\psi}\right)<0$, while $F_{\frac{1}{\psi}}$ is bounded from above by its behavior at small arguments (see the appendix B, which is non-positive. In consequence, for the case described by Eq. (23) the last term must give a finite contribution to assure existence of a solution at $x>0$ and we find $|\bar{s}|$ to be of necessarily of order $O\left(\frac{D^{\frac{4}{\psi}-4}}{V}\right)$. In a compact way we write our result as:

$$
|\bar{s}|=O\left(\frac{D^{\gamma}}{V}\right) .
$$


Eq. (25) constitutes the essential result of this section.

The key conclusion of the above analysis is that in the limit $L \gg D \gg l_{m i c}$ and for $T \leq T_{c}$ the behavior of $|\bar{s}|$ is controlled by $L$ rather than $D$. Put in other words, if the limit $L \rightarrow \infty$ is performed, keeping $D$ finite, there will be no surviving contribution to $|\bar{s}|$. By virtue of Eq. $(18)$ the same applies to the quantity $\sigma$. This fact opens wide the way to characterize the excess free energy of the system in the scaling limit, which is done in the next section.

It is worth emphasizing that Eq. (25) holds only at criticality and in the low- $T$ phase (for $T \leq T_{c}$ ) and for dimensionality $d$ high enough, namely for $\frac{1}{\psi} \geq \frac{7}{4}$ (corresponding to $d \geq 4$ for $m=1$ ). If the thermodynamic state is fixed above $T_{c}$, the magnitude of $|\bar{s}|$ in the limit $L \gg D \gg l_{\text {mic }}$ is controlled by the distance from the phase transition (measured by the parameter $\varepsilon$ ). On the other hand, for $T \leq T_{c}$, but $\frac{1}{\psi}<\frac{7}{4}|\bar{s}|$ is controlled by $D$ (i.e. $|\bar{s}|$ is finite for $L \rightarrow \infty$ with $D$ finite, but vanishes if $D \rightarrow \infty$ ). In what follows we restrict to the cases, where Eq. (25) holds. For an analysis of the opposite situations see Ref. [12].

\section{Excess grand canonical free energy}

The result of Eq. (25) greatly simplifies the analysis of Eq. (11), leading to the determination of the excess grand canonical free energy $\omega_{s}$. Considering the limit $L \rightarrow \infty$ keeping $D$ finite we may simply put $|\bar{s}| \rightarrow 0^{+}$. We obtain

$$
\omega_{s}=-k_{B} T \frac{\chi^{d-m} \Delta_{m}^{d}}{D^{2 d-m-1}}
$$

where

$$
\Delta_{m}^{d}=\frac{\Gamma(5 / 4)^{\frac{4}{\psi}-1}}{\pi^{\frac{4}{\psi}}} \lim _{\sigma \rightarrow 0^{+}} \sigma^{\frac{4}{\psi}} \sum_{n=1}^{\infty} F_{\frac{1}{\psi}+1}(n \sigma),
$$

and $\chi=\frac{\lambda_{2}^{2}}{\lambda_{1}}$ is temperature-independent length scale. Note that the magnitude of the Casimir interaction may be greatly amplified by manipulating this parameter. Upon putting $\theta_{A}=\frac{1}{2} \mathrm{in} \mathrm{Eq.} \mathrm{(3)} \mathrm{[as} \mathrm{pertinent}$ to the present situation - see e.g. Refs. [6, 26]] and identifying $\Gamma \leftrightarrow \chi^{d-m}$ we match Eq. (3) with the derived formula of Eq. 26, and identify $\Delta_{m}^{d}$ with the scaling function $\Delta(x)$ of Eq. 3 .

Remarkably, Eq. 26) and Eq. 27) hold for arbitrary nonnegative value of the scaling variable $x$ and represent the entire expression for $\omega_{s}$ and not only the asymptotic behavior for $D$ large. The physically relevant new result of the present paper now follows from the analysis of Eq. (27) upon changing dimensionality. As we demonstrate below, the sign of $\Delta_{m}^{d}$ is sensitive to the value of $\frac{1}{\psi}$ and therefore may be varied while manipulating $d$ and $m$. When restricting to the 'uniaxial' case $m=1$, we show that $\Delta_{m}^{d}$ is precisely zero for natural even dimensionalities starting form $d=4$.

We may further simplify Eq. 27) extracting the asymptotic behavior of the function $F$ - see the appendix B. Introducing

$$
G(\kappa)=\int_{0}^{\infty} \mathrm{d} q q^{4 \kappa-5} \phi(q)
$$

we obtain

$$
\Delta_{m}^{d}=4 \zeta\left(\frac{4}{\psi}\right) \frac{\Gamma(5 / 4)^{\frac{4}{\psi}-1}}{\pi^{\frac{4}{\psi}}} G\left(\frac{1}{\psi}+1\right) .
$$

Eq. 29] was already contained in Ref. [12], but its generality and the rich physical consequences encoded in the properties of the function $G$ were completely neglected in that study, which focused 
mostly on the physical dimensionality $d=3$. As we demonstrate below, the function $G(\kappa)$ changes sign for

$$
\kappa=\kappa_{n}=\frac{4 n+7}{4} \text { with } n \in\{0,1,2, \ldots\} .
$$

The fact that $G\left(\kappa=\kappa_{n}\right)=0$ is proven in the appendix B. Below, in Fig. 1 we provide a plot of $G(\kappa)$ evaluated numerically. Particularly interesting is the case $m=1$, where $\frac{1}{\psi}=\frac{d}{2}-\frac{1}{4}$ and the zeroes

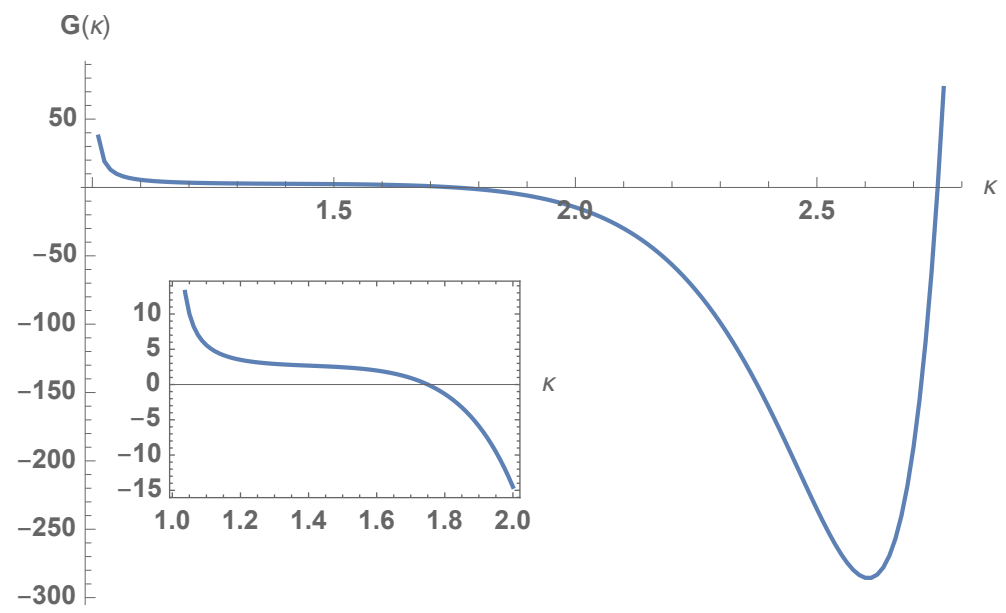

Figure 1: The function $G(\kappa)$ evaluated numerically for a range of arguments capturing its first two zeroes (at $\kappa=\kappa_{0}=\frac{7}{4}$ and $\kappa=\kappa_{1}=\frac{11}{4}$ ). The inset shows the same data in a restricted range of $\kappa$ making the position of $\kappa_{0}$ clearly visible. For increasing values of $\kappa$, the magnitude of oscillations of $G(\kappa)$ rapidly diverges, so that neither $G(\kappa)$ nor its derivative is bounded from above or below.

of $G\left(\frac{1}{\psi}+1\right)$ fall precisely at dimensionality $d=4,6,8, \ldots$ In consequence, the Casimir force is repulsive up to dimensionality $d=4$, attractive for $d \in(4,6)$, repulsive again for $d \in(6,8)$ and so on. The associated Casimir amplitude quickly diverges upon increasing $d$. A remarkable observation concerns the case $d \in\{4,6,8, \ldots\}$ where the entire scaling function $\Delta_{m=1}^{d}$ is strictly zero, and, as we showed above, there is no subleading term surviving the limit $L \rightarrow \infty$.

It is now tempting to speculate about the relevance of the above result beyond $1 / N=0$. By virtue of continuity one does not expect a drastic change of the scaling function when $1 / N$ is varied from zero to an arbitrarily small value $1 / N=\epsilon$, but certainly there is no reason to believe that the dimensionalities marking the boundaries between the attractive and repulsive regimes should still correspond to even natural numbers. Nor are there reasons to expect that the scaling function remains constant upon lifting $1 / N$ from zero. However, considering $1 / N$ arbitrarily small, keeping $x$ fixed and changing $d$, continuity of the scaling function requires that $\Delta_{m=1}^{d}(x)$ changes sign at some $d$ (which may depend on $x$ ).

In Fig. 2 we have illustrated the picture we obtained at $1 / N=0$ together with the anticipated features at $1 / N$ sufficiently small. It is absolutely open, what survives out of this in the physically most interesting cases of $N=3$ and $N=2$, for example whether the dashed lines (boundaries between the repulsive and attractive regimes) emerging from $1 / N=0$ and $d=4,6,8, \ldots$ persist up to $1 / N=0.3(3)$, or (for example) merge in pairs at some values of $1 / N$. Addressing this question is an interesting (presumably challenging) topic for future research. 


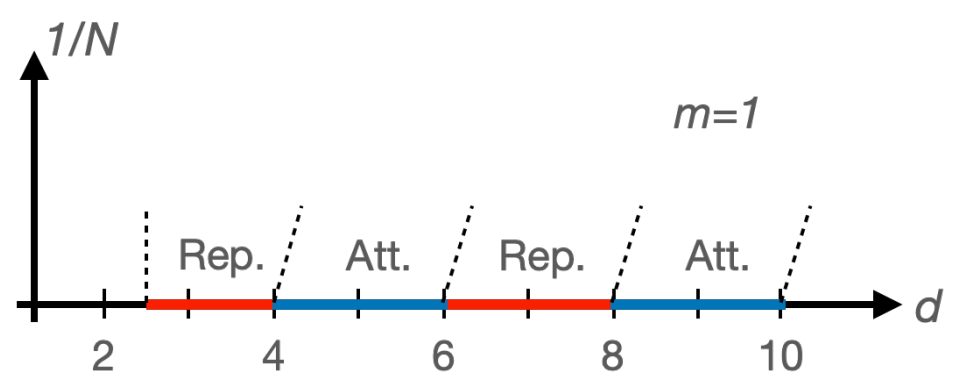

Figure 2: Illustration of the results concerning the sign of the Casimir force for $1 / N=0$ for the uniaxial case $m=1$ together with the expected situation at $1 / N \ll 1$. At $1 / N=0$ the obtained interaction is repulsive for dimensionality $d<4$, attractive for $d \in(4,6)$, repulsive for $d \in(6,8)$ and so on. Occurrence of the boundaries separating the attractive and repulsive regimes (at fixed $x$ - see the main text) is very likely to persist for small $1 / N>0$ but their fate upon increasing $1 / N$ towards $1 / 3$ is completely open.

\section{Discussion and outlook}

In this paper we disclose the surprising properties regarding the sign of the Casimir force in anisotropic system upon varying dimensionality. Employing the imperfect (mean-field) Bose gas as a representative of the (anisotropic) vectorial $N \rightarrow \infty$ class, we demonstrate the periodic alternation of the sign of the Casimir energy upon changing dimensionality. Particularly interesting is the case $m=1$, where the dispersion is quartic in one of the spatial directions and quadratic in the remaining ones. In this situation we demonstrate that the Casimir interaction is repulsive for dimensionality $d \in\left(\frac{5}{2}, 4\right) \cup(6,8) \cup(10,12) \cup \ldots$ and attractive for $d \in(4,6) \cup(8,10) \cup \ldots$. We show moreover, that for $d \in\{4,6,8 \ldots\}$ the Casimir interaction entirely vanishes in the scaling limit. Even though the analysis is performed for a system from the $1 / N=0$ universality class, one may argue that, by continuity, the uncovered unexpected features should also occur for $1 / N$ finite but sufficiently small. The phrase 'sufficiently small' is vague here, and this is by no means excluded that the physically most interesting situations of $N \in\{1,2,3\}$ fall into this category. A clarification of this issue requires further studies e.g. from the point of view of the field-theoretic approaches with $1 / N$ expansion.

The possibility of modifying the sign of Casimir-type forces (for example by manipulating the boundary fields) was recently considered in a number of contexts [27-36]. The presently analyzed setup predicting their actual vanishing appears however entirely new and somewhat surprising.

Our predictions are certainly open to verification by numerical simulations. Restricting to natural $d$ (where simulations are usually performed) we then expect a repulsive interaction at $d=3$ and attractive for $d=5$, while the obtained force should entirely vanish in the vicinity of $d=4$ (again provided $1 / N$ is sufficiently small).

Another context, where our study may be of relevance concerns quantum phase transitions. The present analysis is performed in the vicinity (or below) a thermal phase transition, where $T$ is assumed finite. Technically this means that $D \gg \lambda_{1,2}$, which forbids taking the $T \rightarrow 0$ limit. It is however recognized, that (at least bulk) scaling properties of a system in the vicinity of many thermal phase transitions are closely related to those of a quantum (i.e. occurring at $T=0$ and therefore driven by quantum fluctuations) phase transition in elevated dimensionality $d_{q}=d+z$. [37] This equivalence was in particular demonstrated for the isotropic variant of the present model. [38] To which extent 
the quantum-classical correspondence also holds for anisotropic systems and for interfacial properties needs clarifying studies. Assuming such an extension is possible, the peculiar case of $d_{q}=4$, where the Casimir interaction vanishes should relate to physical dimensionality $d=3$ for systems with a gap in the ordered phase (where $z=1$ ). The celebrated example is the quantum Ising model in a transverse field. Its classical counterpart may realize a Lifshitz point upon introducing the next-nearest-neighbor interaction (the so-called ANNNI model). [39] On the other hand, interacting bosons are characterized by $z=2$, which makes both $d_{q}=4$ and $d_{q}=5$ ( $d=2$ and $d=3$ respectively) physically conceivable in anisotropic optical lattice systems. [40,41]

\section{Acknowledgements}

We are grateful to Marek Napiórkowski and Piotr Nowakowski for discussions as well as reading the manuscript and providing helpful suggestions.

Funding information PJ acknowledges support from the Polish National Science Center via 2017/26/E/ST3/00211.

\section{A The remainder term $\mathcal{R}_{1}^{(1)}$}

In this appendix we provide a discussion of the remainder term $\mathcal{R}_{1}^{(1)}$ occurring in Eq. 17 . This arises [12] while approximating the sum $\sum_{r=1}^{\infty} f(r)$ with the integral $\int_{0}^{\infty} f(r)$ for

$$
f(r)=e^{r \bar{s}} r^{-\frac{1}{\psi}} \phi\left(C_{n} r^{-\frac{1}{4}}\right)
$$

and $C_{n}=\frac{\pi n}{\Gamma(5 / 4)} \frac{D}{\lambda_{2}}$ with $n \in \mathbb{N}$. The Euler-Maclaurin formula for our case may be written as

$$
\begin{aligned}
\sum_{r=1}^{M} f(r)-\int_{0}^{M} \mathrm{~d} r f(r) & =\frac{f(M)-f(0)}{2}+ \\
& \sum_{k=1}^{[p / 2]} \frac{b_{2 k}}{(2 k) !}\left(f^{(2 k-1)}(M)-f^{(2 k-1)}(0)\right)+\mathcal{R}_{p},
\end{aligned}
$$

with $M \rightarrow \infty, p \in \mathbb{N}$ arbitrary, $[p / 2]$ denoting the integer part of $p / 2, b_{2 k}$ being numerical coefficients of no relevance here, and finally

$$
\mathcal{R}_{p}=(-1)^{p+1} \frac{1}{p !} \int_{0}^{M} \mathrm{~d} r f^{(p)}(r) P_{p}(r) .
$$

Here $P_{p}(r)$ are the periodized Bernoulli functions. The asymptotic forms of the function $\phi(x)$ are given in Ref. [42]. For the present analysis it is sufficient to know that $\phi(x=0)=$ const and can be expanded in powers of $x$ in the neighborhood of $x=0$, while $\left|\phi\left(C_{n} r^{-1 / 4}\right)\right| \approx r^{\gamma} e^{-\alpha / r^{1 / 3}}$ for $r \rightarrow 0^{+}$with $\gamma$ and $\alpha$ positive. Using these forms for $r \ll 1$ and $r \gg 1$ in the definition of $f(r)$ one finds that $f(r)$ vanishes at $r \rightarrow 0$ and $r \rightarrow \infty$ together with all of its derivatives. This is true for any values of $D$. In consequence, $\mathcal{R}_{p}$ is the only nonzero component on the RHS of Eq. (32). By choosing $p=1$ and recalling that $P_{1}(r)=(r-[r])-\frac{1}{2}$, we obtain that

$$
\sum_{r=1}^{M} f(r)-\int_{0}^{M} \mathrm{~d} r f(r)=\mathcal{R}_{1}=\int_{0}^{\infty} \mathrm{d} r\left[\frac{d}{d r}\left(\frac{e^{r \bar{s}}}{r^{1 / \psi}} \phi\left(C_{n} r^{-1 / 4}\right)\right)\right] P_{1}(r) .
$$


We now introduce $x=r|\bar{s}|$, and write $\mathcal{R}_{1}$ as

$$
\mathcal{R}_{1}=|\bar{s}|^{1 / \psi} \int_{0}^{\infty} \mathrm{d} x\left[\frac{d}{d x}\left(\frac{e^{-x}}{x^{1 / \psi}} \phi\left(\frac{n \sigma}{x^{1 / 4}}\right)\right)\right]\left\{\frac{x}{|\bar{s}|}-\left[\frac{x}{|\bar{s}|}\right]-\frac{1}{2}\right\} .
$$

The integral is convergent for any $\bar{s}$, and therefore $\left|\mathcal{R}_{1}\right| \leq O\left(|\bar{s}|^{1 / \psi}\right)$, which is already sufficient to justify dropping the remainder in Eq. (17). The bound we used is very crude, and in fact the integral vanishes for $|\bar{s}| \rightarrow 0$ due to the violent oscillations of the term $\left\{\frac{x}{|\bar{s}|}-\left[\frac{x}{|\bar{s}|}\right]-\frac{1}{2}\right\}$.

\section{B Properties of $F$ and $G$ functions}

In this appendix we exhibit the relevant properties of the functions $F$ and $G$. In particular we demonstrate the zeroes of $G$.

The function $F_{K}(x)$ is defined in Eq. (18) and a change of variables brings it to the form

$$
F_{\kappa}(x)=\int_{0}^{\infty} \mathrm{d} p \frac{e^{-p}}{p^{\kappa}} \phi\left(\frac{x}{p^{1 / 4}}\right)=\frac{4}{x^{4 \kappa-4}} \int_{0}^{\infty} \mathrm{d} q q^{4 \kappa-5} e^{-\frac{x^{4}}{q^{4}}} \phi(q) .
$$

We are interested here only in $\kappa>1$ and sufficiently small $x$. The integrand on the RHS of Eq. (36) is exponentially suppressed for $q \ll x$, while for $q \gg x$ the term $e^{-\frac{x^{4}}{q^{4}}}$ may be approximated by unity. Since $\phi(q)$ is constant for $q \rightarrow 0$, we may replace $e^{-\frac{x^{4}}{q^{4}}} \rightarrow 1$ for $x \ll 1$. We obtain

$$
F_{\kappa}(x \approx 0) \approx \frac{4}{x^{4 \kappa-4}} G(\kappa)+\ldots,
$$

where the function $G(\kappa)$ is defined by Eq. 28 . We have checked that for $\kappa \in\left[\frac{7}{4}, 2\right) F_{\kappa}(x)$ is a decreasing function of $x$, bounded from above by its behavior near $x=0$.

We now demonstrate that $G\left(\kappa=\kappa_{n}\right)=0$ for $\kappa_{n}=\frac{4 n+7}{4}$ and $n \in\{0,1,2, \ldots\}$. Plugging $\kappa=\kappa_{n}$ into the definition of $G(\kappa)$ and using $\phi(-q)=\phi(q)$ we change the order of the integrals occurring in $G(\kappa)$ and obtain:

$$
G\left(\kappa_{n}\right)=\frac{1}{2} \int_{-\infty}^{\infty} \mathrm{d} x e^{-x^{4}} \int_{-\infty}^{\infty} \mathrm{d} q q^{4 n+2} e^{i q x} .
$$

We now use the representation of the $l$-th derivative of Dirac delta:

$$
\delta^{(l)}(x)=\frac{1}{2 \pi} \int_{-\infty}^{\infty} \mathrm{d} q(i q)^{l} e^{i q x},
$$

which leads to

$$
G\left(\kappa_{n}\right)=(-i)^{4 n+2} \pi \int_{-\infty}^{\infty} \mathrm{d} x \delta^{(4 n+2)}(x) e^{-x^{4}} .
$$

Considering that $\int_{-\infty}^{\infty} \mathrm{d} x \delta^{(l)}(x) f(x)=(-1)^{l} f^{(l)}(0)$, we find:

$$
G\left(\kappa_{n}\right)=\left.\pi(i)^{4 n+2}\left(e^{-x^{4}}\right)^{(4 n+2)}\right|_{x=0}=0 .
$$

In performing the last step we observed, that only the 4-th, 8-th, 12-th and so on derivatives of the function $f(x)=e^{-x^{4}}$ are nonvanishing at $x=0$. 


\section{References}

[1] V. M. Mostepanenko and N. N. Trunov, The Casimir effect and its applications, Soviet Physics Uspekhi 31(11), 965 (1988), doi:10.1070/pu1988v031n11abeh005641.

[2] M. Krech, The Casimir Effect in Critical Systems, WORLD SCIENTIFIC, doi:10.1142/2434 (1994).

[3] M. Kardar and R. Golestanian, The "friction" of vacuum, and other fluctuation-induced forces, Rev. Mod. Phys. 71, 1233 (1999), doi:10.1103/RevModPhys.71.1233.

[4] J. G. Brankov, D. M. Danchev and N. S. Tonchev, Theory of Critical Phenomena in Finite-Size Systems, WORLD SCIENTIFIC, doi:10.1142/4146(2000).

[5] A. Maciołek and S. Dietrich, Collective behavior of colloids due to critical Casimir interactions, Rev. Mod. Phys. 90, 045001 (2018), doi:10.1103/RevModPhys.90.045001.

[6] H. W. Diehl, Critical behavior at m-axial Lifshitz points, Acta Physica Slovaca 52, 271 (2002).

[7] M. Burgsmüller, H. W. Diehl and M. A. Shpot, Fluctuation-induced forces in strongly anisotropic critical systems, Journal of Statistical Mechanics: Theory and Experiment 2010(11), P11020 (2010), doi:10.1088/1742-5468/2010/11/p11020.

[8] R. M. Hornreich, M. Luban and S. Shtrikman, Critical behavior at the onset of $\overrightarrow{\mathrm{k}}-$ space instability on the $\lambda$ line, Phys. Rev. Lett. 35, 1678 (1975), doi:10.1103/PhysRevLett.35.1678.

[9] P. M. Chaikin and T. C. Lubensky, Principles of condensed matter physics, Cambridge University Press (1995).

[10] X.-z. Li, H.-b. Cheng, J.-m. Li and X.-h. Zhai, Attractive or repulsive nature of the Casimir force for rectangular cavity, Phys. Rev. D 56, 2155 (1997), doi:10.1103/PhysRevD.56.2155.

[11] O. Kenneth and I. Klich, Opposites Attract: A Theorem about the Casimir Force, Phys. Rev. Lett. 97, 160401 (2006), doi:10.1103/PhysRevLett.97.160401.

[12] M. Łebek and P. Jakubczyk, Dimensional crossovers and Casimir forces for the Bose gas in anisotropic optical lattices, Phys. Rev. A 102, 013324 (2020), doi: 10.1103/PhysRevA.102.013324.

[13] H. W. Diehl and S. B. Rutkevich, Fluctuation-induced forces in confined ideal and imperfect Bose gases, Phys. Rev. E 95, 062112 (2017), doi:10.1103/PhysRevE.95.062112.

[14] D. Danchev, Finite-size scaling Casimir force function: Exact spherical-model results, Phys. Rev. E 53, 2104 (1996), doi:10.1103/PhysRevE.53.2104.

[15] D. Dantchev and M. Krech, Critical Casimir force and its fluctuations in lattice spin models: Exact and Monte Carlo results, Phys. Rev. E 69(4), 046119 (2004), doi:10.1103/PhysRevE.69.046119.

[16] E. B. Davies, The thermodynamic limit for an imperfect Boson gas, Comm. Math. Phys. 28(1), 69 (1972). 
[17] E. Buffet and J. V. Pulè, Fluctuation properties of the imperfect Bose gas, Journal of Mathematical Physics 24(6), 1608 (1983), doi:10.1063/1.525855, https://doi.org/10.1063/1. 525855 .

[18] V. A. Zagrebnov and J.-B. Bru, The Bogoliubov model of weakly imperfect Bose gas, Physics Reports 350(5), 291 (2001), doi:https://doi.org/10.1016/S0370-1573(00)00132-0.

[19] M. Napiórkowski and J. Piasecki, Casimir force induced by an imperfect Bose gas, Phys. Rev. E 84, 061105 (2011), doi:10.1103/PhysRevE.84.061105

[20] M. Napiórkowski, P. Jakubczyk and K. Nowak, The imperfect Bose gas in d dimensions: critical behavior and Casimir forces, Journal of Statistical Mechanics: Theory and Experiment 2013(06), P06015 (2013), doi:10.1088/1742-5468/2013/06/p06015.

[21] K. Myśliwy and M. Napiórkowski, Thermodynamics of inhomogeneous imperfect quantum gases in harmonic traps, Journal of Statistical Mechanics: Theory and Experiment 2019(6), 063101 (2019), doi:10.1088/1742-5468/ab190d

[22] P. Jakubczyk and J. Wojtkiewicz, Phase diagram and correlation functions of the anisotropic imperfect Bose gas in d dimensions, Journal of Statistical Mechanics: Theory and Experiment 2018(5), 053105 (2018), doi:10.1088/1742-5468/aabc7c

[23] R. M. Ziff, G. E. Uhlenbeck and M. Kac, The ideal Bose-Einstein gas, revisited, Physics Reports 32(4), 169 (1977), doi:https://doi.org/10.1016/0370-1573(77)90052-7.

[24] T. H. Berlin and M. Kac, The Spherical Model of a Ferromagnet, Phys. Rev. 86, 821 (1952), doi: $10.1103 /$ PhysRev.86.821.

[25] H. E. Stanley, Spherical Model as the Limit of Infinite Spin Dimensionality, Phys. Rev. 176, 718 (1968), doi:10.1103/PhysRev.176.718.

[26] Essafi, K., Kownacki, J.-P. and Mouhanna, D., Nonperturbative renormalization group approach to Lifshitz critical behaviour, EPL 98(5), 51002 (2012), doi:10.1209/0295-5075/98/51002.

[27] P. Nowakowski and M. Napiórkowski, Scaling of solvation force in two-dimensional Ising strips, Phys. Rev. E 78, 060602 (2008), doi:10.1103/PhysRevE.78.060602

[28] D. B. Abraham and A. Maciołek, Casimir interactions in ising strips with boundary fields: Exact results, Phys. Rev. Lett. 105, 055701 (2010), doi:10.1103/PhysRevLett.105.055701.

[29] V. Dohm, Critical free energy and Casimir forces in rectangular geometries, Phys. Rev. E 84, 021108 (2011), doi:10.1103/PhysRevE.84.021108.

[30] M. A. Rajabpour, Classification of the sign of the critical Casimir force in twodimensional systems at asymptotically large separations, Phys. Rev. D 94, 105029 (2016), doi:10.1103/PhysRevD.94.105029.

[31] P. Jakubczyk, M. Napiórkowski and T. Sek, Repulsive Casimir forces at quantum criticality, EPL (Europhysics Letters) 113(3), 30006 (2016), doi:10.1209/0295-5075/113/30006

[32] M. Sadhukhan and A. Tkatchenko, Long-Range Repulsion Between Spatially Confined van der Waals Dimers, Phys. Rev. Lett. 118, 210402 (2017), doi:10.1103/PhysRevLett.118.210402. 
[33] A. Flachi, M. Nitta, S. Takada and R. Yoshii, Sign Flip in the Casimir Force for Interacting Fermion Systems, Phys. Rev. Lett. 119, 031601 (2017), doi:10.1103/PhysRevLett.119.031601.

[34] M. M. Faruk and S. Biswas, Repulsive Casimir force in Bose-Einstein Condensate, Journal of Statistical Mechanics: Theory and Experiment 2018(4), 043401 (2018), doi:10.1088/17425468/aab01b.

[35] Y. Voronina, I. Komissarov and K. Sveshnikov, Casimir interactions between two short-range coulomb sources, Annals of Physics 404, 132 (2019), doi:https://doi.org/10.1016/j.aop.2019.02.014.

[36] Y. Voronina, I. Komissarov and K. Sveshnikov, Casimir force variability in one-dimensional QED systems, Phys. Rev. A 99, 062504 (2019), doi:10.1103/PhysRevA.99.062504.

[37] S. Sachdev, Quantum Phase Transitions, Cambridge University Press, 2 edn., doi:10.1017/CBO9780511973765 (2011).

[38] P. Jakubczyk and M. Napiórkowski, Quantum criticality of the imperfect Bose gas in ddimensions, Journal of Statistical Mechanics: Theory and Experiment 2013(10), P10019 (2013), doi: $10.1088 / 1742-5468 / 2013 / 10 / \mathrm{p} 10019$.

[39] W. Selke, The ANNNI model - Theoretical analysis and experimental application, Physics Reports 170(4), 213 (1988), doi:https://doi.org/10.1016/0370-1573(88)90140-8

[40] D. Greif, T. Uehlinger, G. Jotzu, L. Tarruell and T. Esslinger, Short-Range Quantum Magnetism of Ultracold Fermions in an Optical Lattice, Science 340(6138), 1307 (2013), doi: $10.1126 /$ science. 1236362 .

[41] J. Imriška, M. Iazzi, L. Wang, E. Gull, D. Greif, T. Uehlinger, G. Jotzu, L. Tarruell, T. Esslinger and M. Troyer, Thermodynamics and Magnetic Properties of the Anisotropic 3D Hubbard Model, Phys. Rev. Lett. 112, 115301 (2014), doi:10.1103/PhysRevLett.112.115301

[42] J. P. Boyd, The Fourier Transform of the quartic Gaussian exp $\left(-A x^{4}\right)$ : Hypergeometric functions, power series, steepest descent asymptotics and hyperasymptotics and extensions to $\exp \left(-A x^{2 n}\right)$, Applied Mathematics and Computation 241, 75 (2014), doi:https://doi.org/10.1016/j.amc.2014.05.001. 